

\title{
Spectral-domain optical coherence tomography: Removal of autocorrelation using an optical switch
}

Jun Ai and Lihong V. Wang

Citation: Applied Physics Letters 88, 111115 (2006); doi: 10.1063/1.2186520

View online: http://dx.doi.org/10.1063/1.2186520

View Table of Contents: http://scitation.aip.org/content/aip/journal/apl/88/11?ver=pdfcov

Published by the AIP Publishing

\section{Articles you may be interested in}

Speckle contrast diffuse correlation tomography of complex turbid medium flow

Med. Phys. 42, 4000 (2015); 10.1118/1.4922206

Real-time processing for Fourier domain optical coherence tomography using a field programmable gate array

Rev. Sci. Instrum. 79, 114301 (2008); 10.1063/1.3005996

Spectrally resolved bioluminescence tomography using the reciprocity approach

Med. Phys. 35, 4863 (2008); 10.1118/1.2982138

Polyimide amplified piezoelectric scanning mirror for spectral domain optical coherence tomography Appl. Phys. Lett. 89, 263901 (2006); 10.1063/1.2410239

Integrated structural and functional optical imaging combining spectral-domain optical coherence and multiphoton microscopy

Appl. Phys. Lett. 88, 053901 (2006); 10.1063/1.2171477

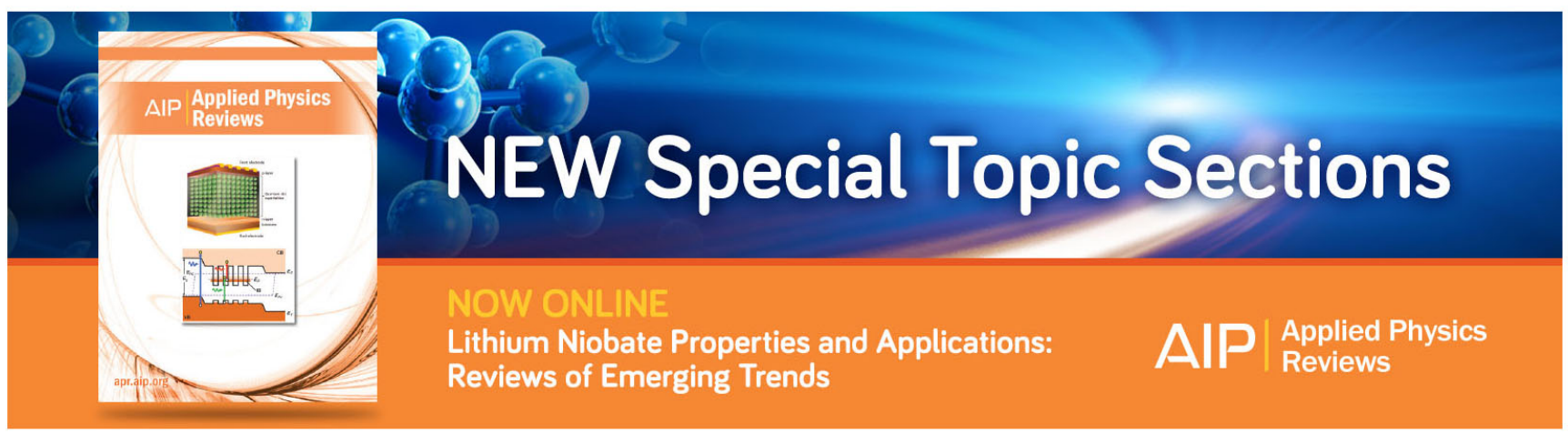




\title{
Spectral-domain optical coherence tomography: Removal of autocorrelation using an optical switch
}

\author{
Jun $\mathrm{Ai}^{\mathrm{a})}$ and Lihong $\mathrm{V}$. Wang ${ }^{\mathrm{b})}$ \\ Optical Imaging Laboratory, Department of Biomedical Engineering, Texas A\&M University, 3120 TAMU, \\ College Station, Texas 77843-3120
}

(Received 21 October 2005; accepted 31 January 2006; published online 15 March 2006)

\begin{abstract}
A novel spectral-domain optical coherence tomography (SD-OCT), in which the self-interferogram of the object wave and the standard SD-OCT interferogram are acquired asynchronously using an optical switch in the reference arm, is presented. After Fourier transformation, the autocorrelation of the object is removed simply by subtraction from the standard SD-OCT image. The measured sensitivity is $103.5 \mathrm{~dB}$ at $z=0.115 \mathrm{~mm}$, and it gradually reduces to $84.1 \mathrm{~dB}$ at $z=3.275 \mathrm{~mm}$. The axial resolution is $15 \mu \mathrm{m}$ in the air. In vivo images of human skin are presented to demonstrate the performance of autocorrelation removal. (C) 2006 American Institute of Physics.
\end{abstract}

[DOI: $10.1063 / 1.2186520$ ]

Frequency-domain or Fourier-domain (FD) optical coherence tomography (OCT) has been demonstrated to offer faster A-scan speed and higher sensitivity than time-domain (TD) OCT. ${ }^{1-9}$ A readout rate of $29 \mathrm{kHz}$ in a line scan charge coupled device (CCD) camera provides an A-scan rate equivalent to hundreds of meters per second, ${ }^{4}$ which easily surpasses the grating-lens-based rapid scanning optical delay line that is widely used in high-speed TD-OCT. There are two primary types of FD-OCT implementations, depending on the source and detector configuration. The first type, i.e., the spectral domain (SD) OCT, is based on low coherence source and spectrometer/array detector configuration. The second type FD-OCT utilizes a wavelength swept source and a single detector. ${ }^{9}$

The challenge in FD-OCT is the inherent autocorrelation, which not only obscures the image interpretation but also degrades the system sensitivity. To reduce the autocorrelation, a phase-shifting technique has been demonstrated, which requires 2-5 consecutive acquisitions of FD-OCT interferograms, each with a precise phase shift, via a piezoelectric controlled translation stage or an electro-optical phase modulator in the reference arm. ${ }^{4-8}$

Recently, we developed a technique of self-elimination of autocorrelation (SEA) and demonstrated a SEA FD-OCT system, ${ }^{1}$ in which, a two-dimensional array CCD camera was used to simultaneously record both the two-arm interferogram $\operatorname{ro}(k)$ and the sole object-arm interferogram obj $(k)$, where $k$ is the wave number. The two interferograms were then inversely Fourier transformed to the spatial counterparts $\operatorname{RO}(z)$ and $\operatorname{OBJ}(z)$, where $z$ is the spatial coordinate of the object with respect to the reference plane. The object autocorrelation $\mathrm{OBJ}(z)$ is eliminated simply by subtraction. Since the SEA requires neither phase shifting nor calculation of the object complex spectrum, it reduces computational resources and eradicates potential problems such as polychromatic phase error associated with the phase-shifting technique.

In this letter, we report on a novel SEA SD-OCT system, in which an optical switch is placed in the reference arm and

\footnotetext{
${ }^{a}$ Present address: Physical Optics Corporation, Torrance, CA; electronic mail: jun8ai@yahoo.ca



a one-dimensional line scan CCD camera is used to record $\operatorname{ro}(k)$ and obj $(k)$ by turning on and off the switch asynchronously. Compared with Ref. 1 , the new system has no beamsplitter in the object arm and therefore eradicates the $6 \mathrm{~dB}$ extra loss (double-pass). Besides, the line scan CCD camera costs only $20 \%$ of the 2D CCD camera used in Ref. 1. Furthermore, the line scan camera can provide an as fast as $29 \mathrm{kHz}$ line rate, offering potentials for real-time SEA SDOCT.

The procedure of SEA technique comprises: ${ }^{1}$ (1) Acquire $\operatorname{ro}(k), \operatorname{obj}(k)$, and $\operatorname{ref}(k)$; (2) Fourier transform them to $\operatorname{RO}(z), \operatorname{OBJ}(z)$, and $\operatorname{REF}(z)$; and (3) obtain $\mathrm{XC}(z)$ simply by subtracting $\operatorname{OBJ}(z)$ and $\operatorname{REF}(z)$ from $\operatorname{RO}(z)$. In general, the sole reference-arm interferogram $\operatorname{ref}(k)$ needs to be acquired only once by blocking the object arm for the entire image, whereas $\operatorname{ro}(k)$ and $\operatorname{obj}(k)$ have to be acquired for each A-scan.

A schematic diagram of the SD-OCT system is shown in Fig. 1. The light from a superluminescent diode source (central wavelength $850 \mathrm{~nm}$, full width at half maximum $23 \mathrm{~nm}$ ) is split into the reference and object arms by a beam splitter (BS). The power delivered to the object is $0.6 \mathrm{~mW}$. The B scan is provided by a galvanometer mirror (Cambridge $6220 \mathrm{M})$. The backscattered waves from the two arms are recombined by the beam splitter and coupled into a singlemode optical fiber that is attached to a custom-made beam expanding and collimation apparatus (not shown in the diagram) and then enters a custom-designed spectrometer com-

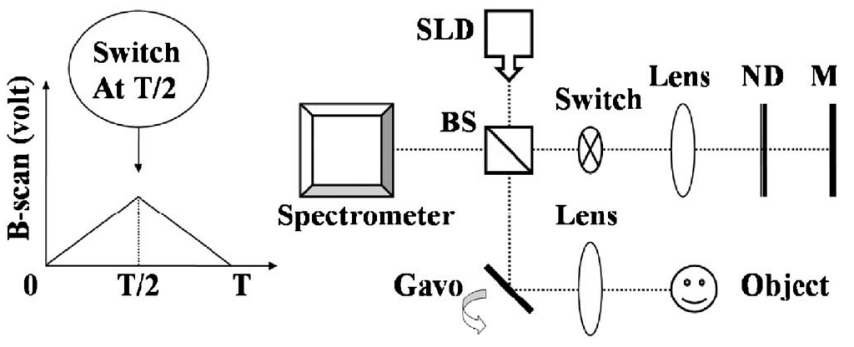

FIG. 1. Schematic of a SEA SD-OCT system. SLD: super-luminescent diode; BS: beam splitter; ND: neutral density filter; $M$ : mirror. The inset shows the B-scan signal of period $T$ for the galvanometer scanner. The switch is turned off at $T / 2$. 


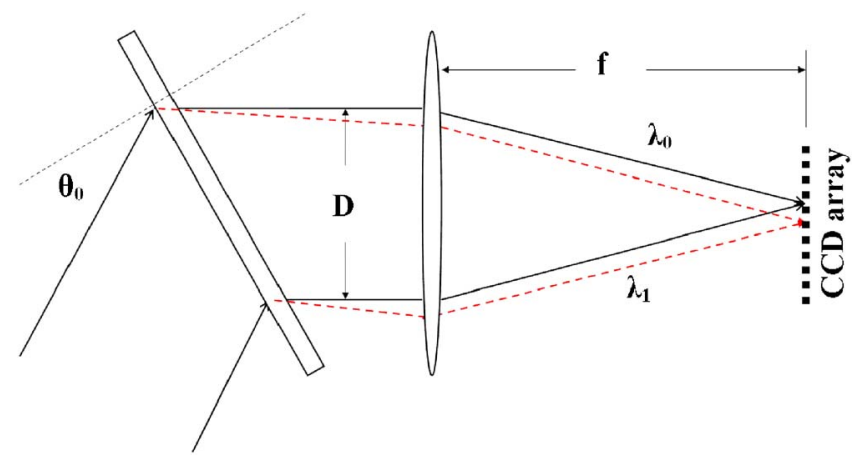

FIG. 2. Diagram of the spectrometer design.

prising a diffraction gratings $\mathrm{G}(1200 \mathrm{lp} / \mathrm{mm}$ volume holographic grating), an achromatic (focal length $200 \mathrm{~mm}$ ), and a line scan CCD camera (ATMEL AVIIVA M2 Camera Link 12-bit $1 \times 2042$ array, pitch $14 \mu \mathrm{m})$. A neutral-density filter (ND) is placed in the reference arm to avoid saturating the CCD camera. The data acquisition of the CCD camera and the operation of the switch and B-scan galvanometer mirror are controlled and synchronized by a Camera-Link board, an analog output board and a custom-developed LabVIEW program.

The inset in Fig. 1 shows a complete cycle $T$ of a triangular signal that drives the B-scan galvanometer mirror. The optical switch (Uniblitz VS14S2T1 shutter) is open during the forward B-scan half cycle for acquisition of $\operatorname{ro}(k)$ and is closed during the backward B-scan half cycle for acquisition of $\operatorname{obj}(k)$. The $\operatorname{ref}(k)$ is acquired only once by blocking the object wave.

Figure 2 shows the diagram of the spectrometer design. The wavelength range dispersed by the aperture of a CCD pixel is determined by $\delta \lambda_{c}=d p \cos \theta /(m f)$, where $p$ is the pitch of the CCD array, $d$ is the grating period, $m$ is the order of diffraction, $\theta$ is the diffraction angle, and $f$ is the focal length of the imaging lens behind the grating. The first order diffraction of the central wavelength $\lambda_{0}$ is aligned with the central pixel of the line scan camera. The blaze angle $\theta_{0}$ is determined by $\sin \theta_{0}=\lambda_{0} /(2 d)$, which is identical as the Bragg condition for a volume holographic grating. The spectral resolution of the diffraction grating is determined by $\delta \lambda_{g}=\lambda d \cos \theta_{0} /(m D)$, where $D$ is the diameter of the collimated beam that is illuminated on the grating. The spectral resolution of the spectrometer is determined by $\delta \lambda=$ the larger of $\left(\delta \lambda_{g}, \delta \lambda_{c}\right)$. The $1 / e^{2}$ Gaussian intensity spot diameter of a laser beam at the back focal plane is determined by $2 \omega=4 \lambda f /(\pi D)$. Optimum spectral resolution and optimum diffraction efficiency are achieved when $\delta \lambda_{g} \leqslant \delta \lambda_{c}$ and $2 \omega$ $\leqslant p$. It is essential that the grating be illuminated by sufficiently wide, uniform, and collimated beam without wave front aberration. Our spectrometer is able to measure a wavelength range of $102.3 \mathrm{~nm}$ with a spectral resolution of $\delta \lambda$ $=50.2 \mathrm{pm}$ at central wavelength $\lambda_{0}=850 \mathrm{~nm}$, which translates to a SD-OCT measurement range of $z_{\max }=3.6 \mathrm{~mm}$ according to $z_{\max }=\lambda_{0}^{2} /(4 \delta \lambda)$.

In our SD-OCT system, the exposure time is chosen as $\tau=1.9 \mathrm{~ms}$ and 524 continuous exposures can be acquired in $T=1$ s. The first 260 exposures are acquired with the optical switch "open," and, therefore, they correspond to ro $(k)$. The last 260 exposures are acquired with the optical switch "closed," and, therefore, they correspond to obj $(k)$. The
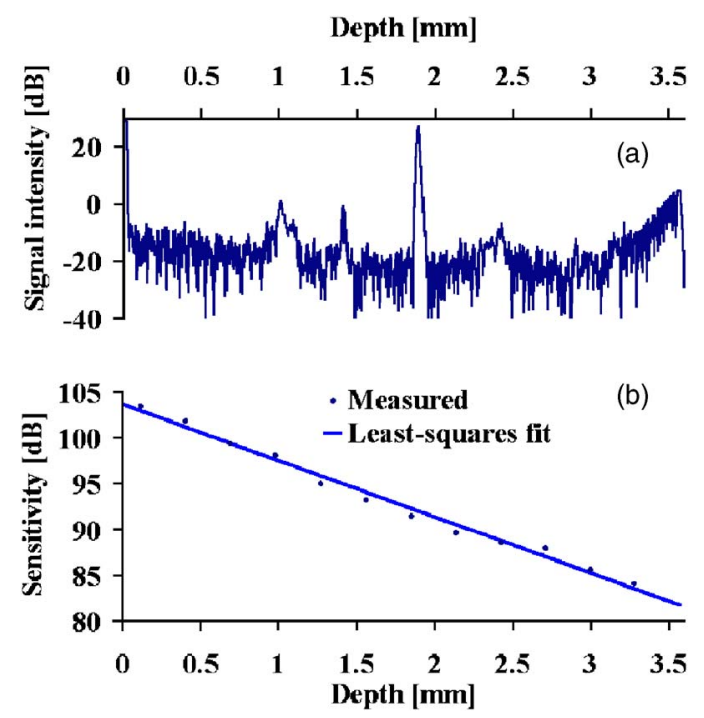

FIG. 3. (a) A typical point spread function, measured at $z=1.85 \mathrm{~mm}$. (b) Sensitivity degradation vs imaging depth $z$. The solid straight line is the least square fit of the measured data points.

transfer time on closing of the switch is $3.0 \mathrm{~ms}$. The 261-264 exposures are excluded because they may be acquired during the transfer time.

The imaging speed of the SEA SD-OCT is 1 frame/s for a frame of $1024 \times 260$ pixels. The line scan rate of the CCD camera can reach $29 \mathrm{kHz}$. The imaging speed of the SEA SD-OCT is constrained by the speed of the optical switch. The object should not move during the cycle of measurement, which is $T=1 \mathrm{~s}$ at present. However, the measurement cycle can be reduced drastically from $1 \mathrm{~s}$ to $524 / 29 \mathrm{kHz}$ $=18 \mathrm{~ms}$, provided a fast optical switch with a transfer time of $\sim 50 \mu \mathrm{s}$ is used. Nevertheless, our experiments show the present system is capable of in vivo imaging of human skin.

The single-pass attenuation of the neutral density filter is measured to be $23.5 \mathrm{~dB}$. Figure 3(a) shows a typical point spread function, measured at $z=1.85 \mathrm{~mm}$. The signal-tonoise ratio is about $44.5 \mathrm{~dB}$, which adding the double-pass insertion loss of the neutral density filter, gives a sensitivity of $91.5 \mathrm{~dB}$.

Figure 3(b) shows the measured sensitivity degradation versus imaging depth $z$. The solid straight line is the least square fit of the measured data points. The measured sensitivity is $103.5 \mathrm{~dB}$ at $0.115 \mathrm{~mm}$, and it gradually reduces to $84.1 \mathrm{~dB}$ at $3.275 \mathrm{~mm}$. The decrease in sensitivity with depth is expected because the finite spectral resolution of the spectrometer reduces fringe visibility at higher fringe frequencies. ${ }^{10,11}$ The slope of the line gives a sensitivity degradation rate of $-6.1 \mathrm{~dB} / \mathrm{mm}$. As a result, the object should be placed closer to the reference plane to achieve optimum sensitivity. However, the autocorrelation also becomes stronger when the object is closer to the reference plane. In our SEA SD-OCT, the autocorrelation artifacts of the source and the object are simply removed by SEA. Therefore, the object can be placed close to the reference plane to achieve optimum imaging performance.

The capability of SEA of our SD-OCT system is validated by imaging a U.S. Air Force 1951 positive target in a way similar to what we did in Ref. 1 . The axial resolution is $15 \mu \mathrm{m}$ measured at $z=0.115 \mathrm{~mm}$ in the air. The SEA SDOCT system is then used for imaging biological tissues. Figure $4(\mathrm{a})$ is an in vivo standard SD-OCT image, i.e., $\mathrm{RO}(z)$ of 



FIG. 4. (a) Standard SD-OCT and (b) SEA SD-OCT images of in vivo human skin. The lateral B scan covers $3 \mathrm{~mm}$. The scale bar represents $0.3 \mathrm{~mm}$.

the ventral portion of a human skin obtained from the SD-OCT system. The image is plotted in the logarithmic intensity decibel scale and shows the beginning 600 axial and all 260 transverse pixels. The remaining 601-1024 axial pixels are not shown because they reveal no structure of the skin. The focal plane is positioned in the middle of the depth range. The horizontal high intensity line at the top $(z=0)$ is contributed by the dc components of the autocorrelation of the reference and the autocorrelation of the object. In addition, the ac components of the object autocorrelation are spread from near $z=0$ down to deep inside the skin, which degrade the signal-to-noise ratio of the image and obscure the internal structure of the skin. In comparison, Fig. 4(b) shows $\mathrm{XC}(z)$, the SEA SD-OCT image of the skin, obtained by subtracting $\operatorname{OBJ}(z)$ and $\operatorname{REF}(z)$ from $\operatorname{RO}(z)$. It is clear that the autocorrelation interference is eliminated completely, and, as a result, the image quality of the internal structure of the skin is enhanced dramatically.

In conclusion, we have developed an autocorrelation self-elimination spectral-domain optical coherence tomography using an optical switch in the reference arm. The measured sensitivity is $103.5 \mathrm{~dB}$ at $z=0.115 \mathrm{~mm}$, and it gradually reduces to $84.1 \mathrm{~dB}$ at $z=3.275 \mathrm{~mm}$. The axial resolution is $15 \mu \mathrm{m}$ in the air. The imaging speed of the SEA SD-OCT is $1 \mathrm{frame} / \mathrm{s}$ for a frame of $1024 \times 260$ pixels, with potential to achieve an A-scan rate of $\sim 29 \mathrm{kHz}$ with a faster optical switch. In vivo images of human skin are presented to demonstrate the improvement in the imaging performance after the removal of autocorrelation.

This project is sponsored by the National Institutes of Health Grant No. R01 CA092415.

${ }^{1}$ J. Ai and L. Wang, Opt. Lett. 30, 2939 (2005).

${ }^{2}$ G. Hausler and M. W. Lindner, J. Biomed. Opt. 3, 21 (1998).

${ }^{3}$ S. H. Yun, G. J. Tearney, B. E. Bouma, B. H. Park, and J. F. de Boer, Opt. Express 11, 3598 (2003); http://www.opticsexpress.org.

${ }^{4}$ E. Götzinger, M. Pircher, R. A. Leitgeb, and C. K. Hitzenberger, Opt. Express 13, 583 (2005); http://www.opticsexpress.org.

${ }^{5}$ J. Zhang, J. S. Nelson, and Z. Chen, Opt. Lett. 30, 147 (2005).

${ }^{6}$ R. A. Leitgeb, C. K. Hitzenberger, A. F. Fercher, and T. Bajraszewski, Opt. Lett. 28, 2201 (2003).

${ }^{7}$ J. F. de Boer, B. Cense, B. H. Park, M. C. Pierce, G. J. Tearney, and B. E. Bouma, Opt. Lett. 28, 2067 (2003).

${ }^{8}$ R. Leitgeb, C. K. Hitzenberger, and A. F. Fercher, Opt. Express 11, 889 (2003); http://www.opticsexpress.org.

${ }^{9}$ M. Sarunic, M. Choma, C. Yang, and J. Izatt, Opt. Express 13, 957 (2005); http://www.opticsexpress.org.

${ }^{10}$ C. Dorrer, N. Belabas, J.-P. Likforman, and M. Joffre, J. Opt. Soc. Am. B 17, $1795(2000)$

${ }^{11}$ A. F. Fercher, Opt. Commun. 117, 43 (1995). 